\title{
DE
}

BULGARIAN ACADEMY OF SCIENCES

CYBERNETICS AND INFORMATION TECHNOLOGIES • Volume 14, No 3

Sofia $\bullet 2014$

Print ISSN: 1311-9702; Online ISSN: 1314-4081

DOI: $10.2478 /$ cait-2014-0036

\section{Feedback Linearization Control of the Inertia Wheel Pendulum}

\section{Stanislav Enev}

Faculty of Automatics, Technical University of Sofia, 8 Kl. Ohridski Str., 1000 Sofia, Bulgaria Email: enev@tu-sofia.bg

\begin{abstract}
In this paper, two feedback linearizing control laws for the stabilization of the Inertia Wheel Pendulum are derived: a full-state linearizing controller, generalizing the existing results in literature, with friction ignored in the description and an inputoutput linearizing control law, based on a physically motivated definition of the system output. Experiments are carried out on a laboratory test bed with significant friction in order to test and verify the suggested performance and the results are presented and discussed. The main point to be made as a consequence of the experimental evaluation is the fact that actually the asymptotic stabilization was not achieved, but rather a limit cycling behavior was observed for the full-state linearizing controller. The input-output linearizing controller was able to drive the pendulum to the origin, with the wheel speed settling at a finite value.
\end{abstract}

Keywords: Feedback linearization, inertia wheel pendulum, unstable systems control.

\section{Introduction}

The Inertia Wheel Pendulum (IWP) represents a relatively novel mechanical system attracting a lot of attention in recent years as a research and teaching "tool" in the control systems field. It is a physical pendulum with a rotating symmetrical wheel at the pendulum top and represents an under-actuated mechanical system since only the wheel is actuated while the joint at the pendulum base is not. The IWP dynamics are considered a representative case study when testing and studying different nonlinear control strategies [1-5]. Also, many interesting and not solved control problems arise in under-actuated configurations (such as control in robot walking, running, flying and so on), and being an under-actuated system, the IWP may be useful when trying to gain insight into the dynamics involved and the 
possible ways to control them. From a pedagogical viewpoint, it is attractive mainly because it is "the most linear" of all pendulum systems, having only one nonlinear term in its motion equations (the gravity induced torque on the pendulum), which makes it suitable for introducing unstable systems and state-space techniques in linear control courses.

It is also a nonlinear, but full-state feedback linearizable system when ignoring damping in the dynamics, i.e a flat output can be constructed, so that the relative degree of the system equals the dimension of the state space. In [1], one of the first papers, dealing with the control problem of the IWP, a full-state linearizing controller is designed for stabilization at the unstable equilibrium point, corresponding to the inverted position of the pendulum.

In this paper two feedback linearization control designs are presented for stabilizing the IWP at the unstable equilibrium, i.e., two balancing controllers are designed. The first one represents generalization of the one, proposed in [1], including also the wheel angle in the description of the system. As in [1], no damping is accounted for in the description. The experimental results showed however, that the friction at the pendulum joint has a great impact on the achieved asymptotic behavior of the system. On the other hand, firstly, as mentioned in [3] and [6], if even only a viscous friction damping is added to the description, the flatness of the system is lost and it is no longer full-state linearizable (it is easily shown that if a viscous damping term is added, the suggested output definition in [1] no longer leads to full-state linearization and in fact, a flat output for the system does not exist). Secondly, since the friction forces present a discontinuous behavior and most friction models [7] capture this, the derivation-based technique that generates the linearizing transformation and control law becomes intractable and suggests no way to compensate the friction terms. A second linearizing controller is designed, using a physically motivated definition of the system output, achieving only an input-output linearization. This design enables also to incorporate easily a friction compensation term in the control law. The properties of the resulting internal dynamics are discussed. Experiments on a laboratory test bed are carried out in order to test the suggested performance for both algorithms.

The rest of the paper is organized as follows. In Section 2 the mathematical modeling of IWP is given. In Section 3, the two feedback linearizing transformations and control laws are derived. In Section 4, the laboratory test bed is described and the obtained experimental results are presented. Finally, the last section is devoted to summary and discussion of the obtained results.

\section{Mathematical modeling of the IWP}

The mechanical structure of the IWP is schematically depicted in Fig. 1.

The following variable and parameter notations are used:

$\theta_{1}(t)$ - pendulum angle;

$\theta_{2}(t)$ - wheel angle (both, as defined in Fig. 1; for the wheel angle, the zero

position can be taken arbitrary); 
$\tau(t)$ - torque acting between the wheel and pendulum (created by an actuator mounted on the rod's end);

$m_{11}$ - mass of the rod;

$m_{12}$ - mass of the actuator's fixed part;

$m_{2}$ - mass of the inertia wheel plus the mass of actuator's rotating part;

$l_{1}$ - distance to the center of mass of the rod;

$l_{2}$ - distance to the center of mass of the wheel (the wheel and the rotor being symmetrical, this is actually the distance to the wheel axis);

$J_{1}$ - the moment of inertia of the pendulum around its center of mass;

$J_{2}$ - the moment of inertia of the wheel (plus actuator's rotor).

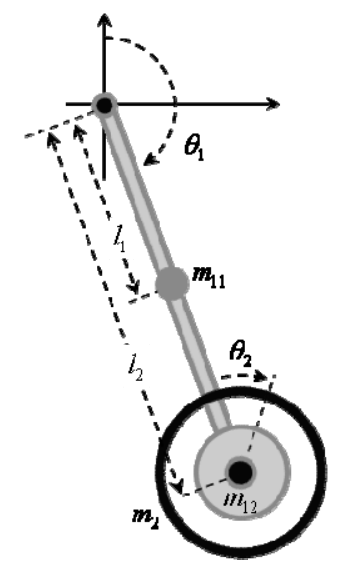

Fig. 1. Inertia wheel pendulum

Assuming that the axis of rotation of the wheel is parallel to the pendulum axis, both perpendicular to a vertical plane, and the wheel is symmetric with respect to its axis of rotation, the Lagrangian is written as

$$
L=V-U \text {, }
$$

with: $V=\frac{1}{2}\left(m_{11} l_{1}^{2} \dot{\theta}_{1}^{2}+\left(m_{12}+m_{2}\right) l_{2}^{2} \dot{\theta}_{2}^{2}+J_{1} \dot{\theta}_{1}^{2}+J_{2}\left(\dot{\theta}_{1}+\dot{\theta}_{2}\right)^{2}\right), U=m_{c} g\left(1+\cos \theta_{1}\right)$, $m_{c}=m_{11} l_{1}+\left(m_{12}+m_{2}\right) l_{2}$ and $g$ being the gravity acceleration.

The Euler-Lagrange equations of motion are easily obtained as

$$
\frac{d}{d t} \frac{\partial L}{\partial \dot{\theta}_{1}}-\frac{\partial L}{\partial \theta_{1}}=0 \text { and } \frac{d}{d t} \frac{\partial L}{\partial \dot{\theta}_{2}}-\frac{\partial L}{\partial \theta_{2}}=\tau
$$

and can be put in the following form:

$$
\begin{aligned}
& \left(m_{11} l_{1}^{2}+\left(m_{12}+m_{2}\right) l_{2}^{2}+J_{1}+J_{2}\right) \ddot{\theta}_{1}+J_{2} \ddot{\theta}_{2}=m_{c} g \sin \theta_{1} . \\
& J_{2} \ddot{\theta}_{1}+J_{2} \ddot{\theta}_{2}=\tau
\end{aligned}
$$

A new term $-f_{p}\left(\dot{\theta}_{1}, \cdot\right)$ is added to the right-hand side of the first equation in (2) to account for the friction in the pendulum joint. 


\section{Feedback linearization based control law design}

The equations of motion of the IWP - (2) are first put in the following form:

$$
\left[\begin{array}{l}
\ddot{\theta}_{1} \\
\ddot{\theta}_{2}
\end{array}\right]=\left[\begin{array}{ll}
a_{11} & a_{12} \\
a_{21} & a_{22}
\end{array}\right]\left[\begin{array}{c}
m_{c} g \sin \theta_{1}-f_{p} \\
\tau
\end{array}\right],
$$

with

$$
\begin{gathered}
a_{11}=J_{2} / D, a_{12}=a_{21}=-J_{2} / D, \\
a_{22}=\left(m_{11} l_{1}^{2}+\left(m_{12}+m_{2}\right) l_{2}^{2}+J_{1}+J_{2}\right) / D, D=\left(m_{11} l_{1}^{2}+\left(m_{12}+m_{2}\right) l_{2}^{2}+J_{1}\right) J_{2} .
\end{gathered}
$$

A state space model is written from (3), with state variables $\left[x_{1}, x_{2}, x_{3}, x_{4}\right]^{\mathrm{T}} \equiv\left[\theta_{1}, \dot{\theta}_{1}, \theta_{2}, \dot{\theta}_{2}\right]^{\mathrm{T}}$ as

$$
\dot{\boldsymbol{x}}=\boldsymbol{f}(\boldsymbol{x})+\boldsymbol{g} \tau \text {, with } \boldsymbol{f}(\boldsymbol{x})=\left[\begin{array}{c}
x_{2} \\
a_{11} m_{c} g \sin \theta_{1}-a_{11} f_{p} \\
x_{4} \\
a_{21} m_{c} g \sin \theta_{1}-a_{21} f_{p}
\end{array}\right], \boldsymbol{g}=\left[\begin{array}{c}
0 \\
a_{12} \\
0 \\
a_{22}
\end{array}\right] \text {. }
$$

Two linearizing state transformations and control laws for the stabilization of the IWP around at the unstable equilibrium point are derived using model (4). The first one, leading to full-state linearization of the system, and the other resulting in partial linearization.

\subsection{Full-state linearization of the IWP}

The full-state linearizing transformation and the control law represent a generalization of the control law proposed in [1], including also the wheel angular position in the description. As suggested there, if the damping term $-f_{p}$ is neglected, defining the output as:

$$
y(\boldsymbol{x})=a_{22} x_{1}+a_{11} x_{3},
$$

leads to a relative degree of the system, equal to the state dimension, thus allowing for full-state linearization.

The new states are obtained by deriving the output function until the input appears in the respective expression. We have:

$$
\dot{y}=\frac{\partial y}{\partial \boldsymbol{x}} \dot{\boldsymbol{x}}=\left[a_{22}, 0, a_{11}, 0\right](\boldsymbol{f}(\boldsymbol{x})+\boldsymbol{g} \tau)=\left[a_{22}, 0, a_{11}, 0\right] \boldsymbol{f}(\boldsymbol{x})+\left[a_{22}, 0, a_{11}, 0\right] \boldsymbol{g} \tau \text {. }
$$

The scalar terms $\frac{\partial y}{\partial \boldsymbol{x}} \boldsymbol{f}(\boldsymbol{x})$ and $\frac{\partial y}{\partial \boldsymbol{x}} \boldsymbol{g}$, denoted usually as $L_{f} y$ and $L_{g} y$ respectively, are known as the Lie derivatives of the function $y$ with respect to the vector field $\boldsymbol{f}(\boldsymbol{x})$ and $\boldsymbol{g}$ respectively [8]. We have:

$$
\dot{y}=L_{f} y+L_{g} y \cdot \tau, \text { with } L_{f} y=a_{22} x_{2}+a_{11} x_{4} \text { and } L_{g} y=0 .
$$

For the second-order derivative of the output we have:

$$
\ddot{y}=\frac{\partial \dot{y}}{\partial \boldsymbol{x}} \dot{\boldsymbol{x}}=\frac{\partial \dot{y}}{\partial \boldsymbol{x}}(\boldsymbol{f}(\boldsymbol{x})+\boldsymbol{g} \tau)=\frac{\partial}{\partial \boldsymbol{x}} L_{f} y \cdot(\boldsymbol{f}(\boldsymbol{x})+\boldsymbol{g} \tau)=L_{f}^{2} y+L_{g} L_{f} y \cdot \tau, \text { with }
$$




$$
L_{f}^{2} y=a_{11}\left(a_{22}+a_{21}\right) m_{c} g \sin x_{1} \text { and } L_{g} L_{f} y=0 .
$$

Continuing in the same way, the following is obtained:

$$
\dddot{y}=L_{f}^{3} y+L_{g} L_{f}^{2} y \cdot \tau \text {, with } L_{f}^{3} y=a_{11}\left(a_{22}+a_{21}\right) m_{c} g \cos x_{1} \cdot x_{2} \text { and } L_{g} L_{f}^{2} y=0 .
$$

Finally

$$
\begin{gathered}
y^{(4)}=L_{f}^{4} y+L_{g} L_{f}^{3} y \cdot \tau, \\
L_{f}^{4} y=-a_{11}\left(a_{22}+a_{21}\right) m_{c} g \sin x_{1} \cdot x_{2}^{2}+a_{11}^{2}\left(a_{22}+a_{21}\right) m_{c}^{2} g^{2} \cos x_{1} \sin x_{1}, \\
L_{g} L_{f}^{3} y=a_{12} a_{11}\left(a_{22}+a_{21}\right) m_{c} g \cos x_{1} .
\end{gathered}
$$

The new state vector can be defined as

$$
\boldsymbol{\xi}=\left[\xi_{1}, \xi_{2}, \xi_{3}, \xi_{4}\right]^{\mathrm{T}} \equiv\left[y, L_{f} y, L_{f}^{2} y, L_{f}^{3} y\right]^{\mathrm{T}},,
$$

and the system dynamics are written in terms (partially) of the new coordinates as

$$
\left[\begin{array}{l}
\dot{\xi}_{1} \\
\dot{\xi}_{2} \\
\dot{\xi}_{3} \\
\dot{\xi}_{4}
\end{array}\right]=\left[\begin{array}{c}
\xi_{2} \\
\xi_{3} \\
\xi_{4} \\
L_{f}^{4} y
\end{array}\right]+\left[\begin{array}{c}
0 \\
0 \\
0 \\
L_{g} L_{f}^{3} y
\end{array}\right] \tau .
$$

The fact that (6) represents a valid state transformation can be easily shown by applying the implicit function theorem [8]. It is seen from the expression of $L_{g} L_{f}^{3} y$, that the system, with $y$ as output, has a well defined relative degree for $x_{1} \neq k \frac{\pi}{2}, k=0, \pm 1, \pm 2, \ldots$

Choosing the linearizing control law as

$$
\tau=\frac{1}{L_{g} L_{f}^{3} y}\left(-L_{f}^{4} y+v\right),
$$

with $v$ being the new input variable results in

$$
\left[\begin{array}{l}
\dot{\xi}_{1} \\
\dot{\xi}_{2} \\
\dot{\xi}_{3} \\
\dot{\xi}_{4}
\end{array}\right]=\left[\begin{array}{l}
\xi_{2} \\
\xi_{3} \\
\xi_{4} \\
v
\end{array}\right] .
$$

Thus, the dynamics of the overall system are linearized and rendered equivalent to those of a chain of four integrators (9). The control law can be introduced as long as the relative degree is well defined, which as noted, is the case for $-\frac{\pi}{2}<x_{1}<\frac{\pi}{2}$. This result establishes the control law as useful for the stabilization problem at $\xi=0$.

Now, a linear state feedback control law in an outer control loop can be designed by pole-placement or another linear control technique to generate the new control input $v$ in order to render the closed-loop system stable, that is $v$ can be chosen as 


$$
v=\boldsymbol{K} \boldsymbol{\xi} \text { with } \boldsymbol{K}=\left[k_{1}, k_{2}, k_{3}, k_{4}\right] .
$$

As seen from (6), $\boldsymbol{\xi}=0$ reduces to $x=0$, thus, stabilizing the system around $\boldsymbol{\xi}=0$ results in the stabilization of the IWP at the unstable equilibrium point.

As noted at the beginning of this section, in [1] the same linearizing control law is derived for the reduced model, obtained by excluding the wheel angle from the description (considering it as a cyclic variable), the output function being defined as $\bar{y}(\boldsymbol{x})=a_{22} x_{2}+a_{11} x_{4}=\xi_{2}$. The linearized states, for this output, become $\bar{\xi}=\left[\bar{\xi}_{1}, \bar{\xi}_{2}, \bar{\xi}_{3}\right]^{\mathrm{T}} \equiv\left[\xi_{2}, \xi_{3}, \xi_{4}\right]^{\mathrm{T}}$. When the full model is considered, this output leads to only partial feedback linearization. It is easy to show, that $x_{3}$ is a valid additional coordinate in order to complete the new state vector, so the internal, firstorder dynamics, of the system can be expressed in terms of the wheel angle. Since we have $\dot{x}_{3}=x_{4}$ and, as $\lim _{t \rightarrow \infty} x_{4}(t)=0$ with the outer linear stabilizing controller in place, it is obvious that the internal state remains bounded and converges to a constant value, which in turn depends on the initial conditions.

As mentioned in the introduction, if only a viscous friction term is added, (5) will no longer lead to full-state linearization and actually no such output exists.

\subsection{Input-output linearizing control law of the IWP}

The second control law is derived, starting from a physically meaningful and directly related to the attacked control problem definition of the system output, being the pendulum angle. Formally, we have:

$$
y(\boldsymbol{x})=x_{1} .
$$

The friction term is accounted for in the model. Again, the output function is derived until the input torque appears in the respective expression. We have

$$
\begin{gathered}
\dot{y}=L_{f} y+L_{g} y \cdot \tau, \text { with } L_{f} y=x_{2}, \text { and } L_{g} y=0 ; \\
\ddot{y}=L_{f}^{2} y+L_{g} L_{f} y \cdot \tau \text {, with } L_{f}^{2} y=a_{11} m_{c} g \sin x_{1}-a_{11} f_{p} \text {, and } L_{g} L_{f} y=a_{12} .
\end{gathered}
$$

The linearizing control law is chosen as

$$
\tau=\frac{1}{L_{g} L_{f} y}\left(-L_{f}^{2} y+v\right)=\frac{1}{a_{12}}\left(-a_{11} m_{c} g \sin x_{1}+a_{11} \hat{f}_{p}+v\right),
$$

again, with $v$ being the new input variable, and $\hat{f}_{p}$ being a model of the friction term in the pendulum joint. It is seen that the system, with the pendulum angle as an output, has a well defined relative degree of two, everywhere in the state space. Thus, if $\hat{f}_{p}=f_{p}$, the system is transformed into

$$
\ddot{x}_{1}=v \text {. }
$$

Otherwise, an additional, angular speed feedback term, induced by the friction will appear at the input.

It is easy to show, that actually no coordinate transformation is needed in this case, since $x_{1}$ and $x_{2}$ can be considered as linearized states, and the remaining 
second-order internal dynamics of the system can be expressed in terms of the wheel angle and angular speed $-x_{3}$ and $x_{4}$ respectively.

Again, a linear state feedback control law in an outer control loop can be designed to generate the new control input $v$ in order to render the closed-loop system stable, that is, $v$ can be chosen as: $v=\left[k_{1}, k_{2}\right]\left[x_{1}, x_{2}\right]^{\mathrm{T}}$, with the appropriate values of $k_{1}$ and $k_{2}$.

With (12) in place and taking into account that $a_{21}=-a_{11}$, the internal dynamics of the system is given by:

$$
\begin{gathered}
\dot{x}_{3}=x_{4}, \\
\dot{x}_{4}=\left(a_{12}+a_{22}\right) m_{c} g \sin x_{1}-a_{21} a_{12} f_{p}-a_{22} \hat{f}_{p}+a_{22} a_{12}^{-1} v .
\end{gathered}
$$

It is seen that when $x_{1}$ and $x_{2}$ go to zero with the outer control loop in place, the right-hand side of the second equation in (14) also goes to zero (the input variable $v$ goes to zero, as well as the friction terms). Thus, the wheel speed converges to a constant value, depending on the initial conditions, while evidently the wheel angle diverges.

\section{Laboratory test bed and experimental results}

\subsection{Description of the test bed}

The main blocks of the laboratory test bed are depicted in Fig. 2. It is a hardwarein-the-loop system. The control law is implemented in real-time within the RealTime Windows Target environment of Matlab ${ }^{\circledR}$, with Simulink ${ }^{\circledR}$ being used as control law building environment and an interface for data logging and visualization. The interface to the physical system is realized with the DAQ board NI 6014 through a signal conditioning and power amplification custom-made circuit board.

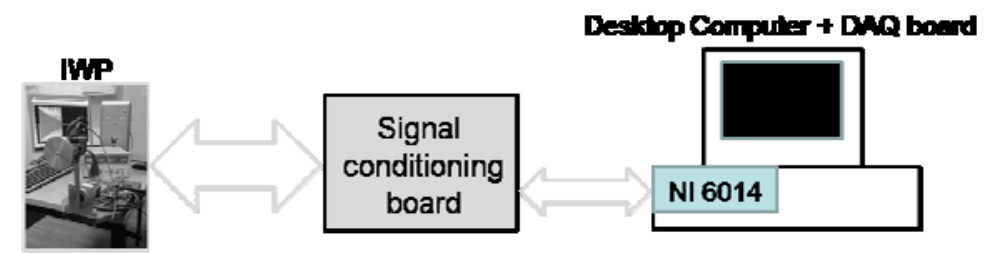

Fig. 2. Block-diagram of the laboratory experiment

The laboratory apparatus is shown in Fig. 3. It comprises a planar physical pendulum made of a rod and the stator of the DC motor, which actuates an inertia wheel, mounted on its rotor. The IWP is instrumented with two incremental quadrature encoders mounted on the two revolute joints of the system. Actually the rod of the pendulum is mounted directly on the shaft of one of the encoders which serves as the pendulum joint of the mechanism (the encoder is fixed to the base plate of the equipment). The pendulum encoder outputs $3600 \mathrm{ppr}$, and the motor encoder outputs 512 ppr. The numbered pieces and devices on Fig. 2 are as follows: 
1 - the inertia wheel; 2 - the DC motor (rated to $24 \mathrm{~V}$ ) with mounted incremental encoder; 3 - the pendulum encoder; 4 - the pendulum rod.

The signal conditioning board contains two blocks.

- A decoder circuit for each of the two incremental encoders used to decode the generated pulse trains. An original decoding circuit realizing combinational and sequential logic, which operates asynchronously with no additional clocking was designed and implemented. Each decoder circuit outputs a direction signal and a pulse, on the second output line, when an edge in any of the encoder two outputs is detected. Thus, the resolution is increased by a factor of 4 up to 14400 counts per revolution for the pendulum encoder and to 2048 for the motor encoder. The decoded signals are fed to the up/down counters of the NI 6014 DAQ board.

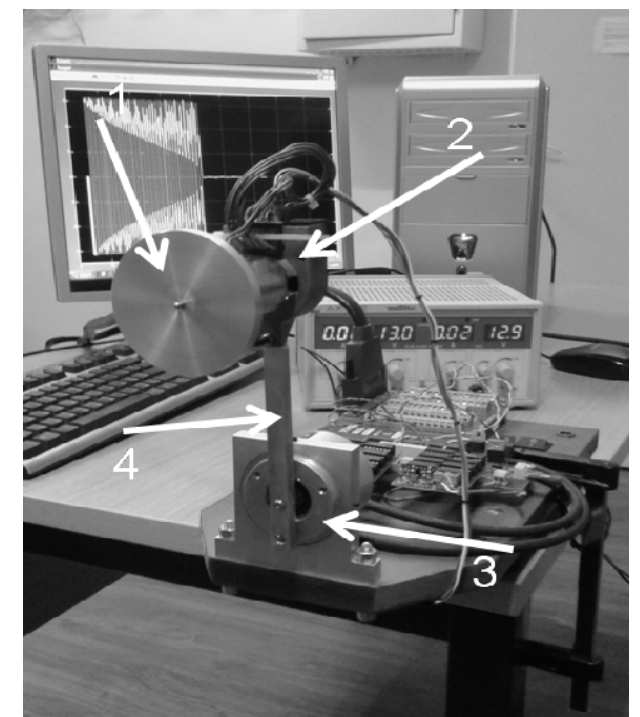

Fig. 3. The IWP balancing at the unstable equilibrium

As a remark we note that using incremental encoders for position sensing on itself requires precise and spot direction change detection. Moreover, vibrations occur when operating the pendulum, which also may lead to interpreting certain counts as "false" or increment the position in the wrong direction. Also, counting only on falling or rising edges may lead to error accumulation in the calculated position value in certain scenarios. These problems were solved with the implemented decoding circuit.

- A linear push-pull outputs circuit with bipolar transistors and flyback diodes providing the high current needed by the motor. The analog low-current control signal, output by the DAQ board, is fed to an operational amplifier with negative feedback on the motor voltage configured for a gain of 1.5. Also, the actual armature voltage of the motor and the voltage drop over a current sensing resistor of $0.1 \Omega$, divided by a resistor divider, are fed to two of the board's analog input lines. The maximum voltage that can be applied to the motor is $15 \mathrm{~V}$, since the analog output of the DAQ board is limited to $10 \mathrm{~V}$. 


\subsection{Model parameters}

The masses of the motor, the wheel and the pendulum rod were measured to be $0.235 \mathrm{~kg}, 0.06 \mathrm{~kg}$ and $0.03 \mathrm{~kg}$ respectively, so $m_{12}+m_{2}=0.295 \mathrm{~kg}$. By design we have: $l_{2}=0.125 \mathrm{~m}$ (the rod is designed to allow also a mounting so that $l_{2}$ is equal to $0.145 \mathrm{~m}$ ) and the distance to the center of mass of the rod with this value of $l_{2}$ is estimated to be around $l_{1}=0.04 \mathrm{~m}$.

The friction model adopted is a static model including Coulomb and viscous friction components [7], so we have: $\hat{f}_{p}\left(\dot{\theta}_{1}\right) \equiv c_{p 1} \operatorname{sign}\left(\dot{\theta}_{1}\right)+c_{p 2} \dot{\theta}_{1}$. As a result of some preliminary identification experiments [9], the following estimates of the rest of the model parameters were found:

$$
J_{1}=180 \times 10^{-6} \mathrm{~kg} . \mathrm{m}^{2}, c_{p 1}=0.00225 \text { (N.m.s) } / \mathrm{rad}, c_{p 2}=0.0002 \text { (N.m.s) } / \mathrm{rad} .
$$

The inertia wheel was designed with a moment of inertia of $32 \times 10^{-6} \mathrm{~kg} \cdot \mathrm{m}^{2}$. A value of $36 \times 10^{-6} \mathrm{~kg} . \mathrm{m}^{2}$ for $J_{2}$ was found to fit better experiments.

The driving torque, produced by the DC motor is given by the following expression:

$$
\tau=k_{\mathrm{m}}\left(u-k_{\mathrm{m}} \dot{\theta}_{2}\right) / R-f_{\mathrm{m}}\left(\dot{\theta}_{2}, \cdot\right),
$$

where $u(t)$ is the armature voltage of the DC motor;

$k_{\mathrm{m}}$ - motor constant (assuming back e.m.f. and torque constants are equal);

$R$ - rotor winding resistance;

$f_{\mathrm{m}}\left(\dot{\theta}_{2}, \cdot\right)$ - friction torque at the rotor bearings and collector brushes.

Again, Coulomb and viscous friction components are adopted and the term is defined as: $f_{\mathrm{m}}\left(\dot{\theta}_{2}\right) \equiv c_{\mathrm{m} 1} \operatorname{sign}\left(\dot{\theta}_{2}\right)+c_{\mathrm{m} 2} \dot{\theta}_{2}$. The inductance of the rotor winding is neglected in the description, since the initial experiments on the laboratory test bed suggested a very small value of the motor electrical time constant.

The following estimates of the model parameters were found in [9] to be:

$$
\begin{gathered}
k_{\mathrm{m}}=0.04(\mathrm{~N} . \mathrm{m}) / \mathrm{A}, R=12.5 \Omega, c_{\mathrm{m} 1}=0.0016(\mathrm{~N} . \mathrm{m} . \mathrm{s}) / \mathrm{rad}, \\
C_{\mathrm{m} 2}=10^{-5}(\mathrm{~N} . \mathrm{m} . \mathrm{s}) / \mathrm{rad} .
\end{gathered}
$$

\subsection{Experimental results}

Two operational aspects of the current laboratory apparatus should be taken in consideration, when analyzing the obtained experimental results. Firstly, significant static friction exists in the pendulum joint, enough to maintain the pendulum still in an upright position in a very small range around the actual vertical position (the direction coinciding with the gravity acceleration direction). The same friction force is able to hold the pendulum deviated from the actual vertical when being down.

Secondly, since incremental encoders are used, a reference point is needed to measure a position. A natural choice for the reference point, which will enable in theory to detect the actual vertical position, is the vertical downward position since it is the stable equilibrium point of the system, and is at a known angular distance to the unstable equilibrium point. Due to the above mentioned friction induced 
deviation of the pendulum from the initial downward vertical position, the measured "zero" value of the pendulum angle also deviates from the exact upright vertical. Moreover, the torque, created by the connecting wires tension also tends to add to this deviation. The same torque acts as a disturbance, when balancing the pendulum around the unstable upright position.

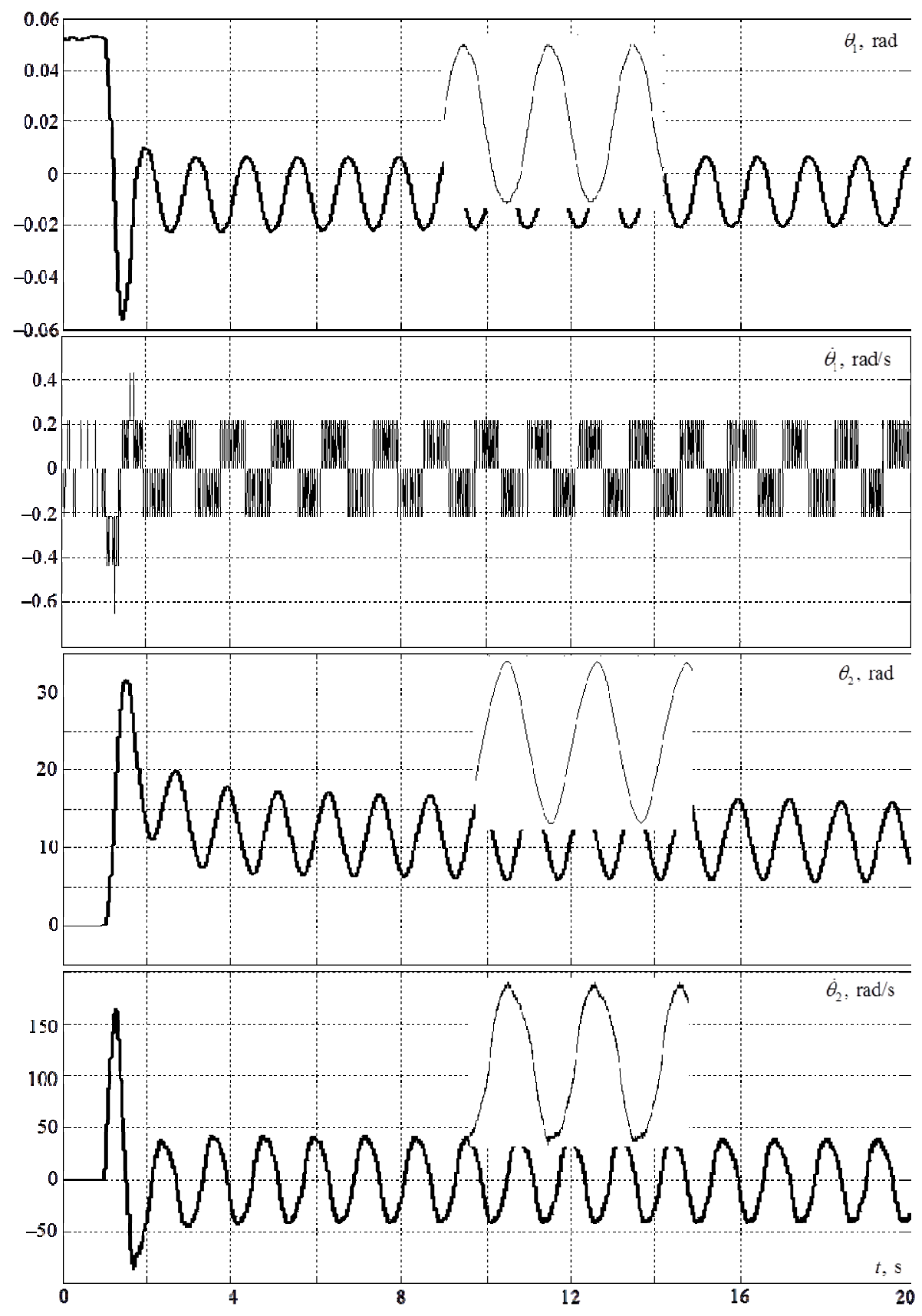

Fig. 4. Experimental trajectories of the IWP coordinates for the full-state linearizing controller 
It should be noted, that no torque saturation is accounted for in the design and the applicability analysis of the proposed control laws so far. Thus, as theoretically the pendulum can be stabilized in the region $-\frac{\pi}{2}<x_{1}<\frac{\pi}{2}$ by using (8) and (10), in practice, this region will be significantly narrower, around the equilibrium point, due to limitations on the available torque.

Finally, if the pendulum angle is considered as a cyclic variable, it should be folded back to the interval $\left(-\frac{\pi}{2} ; \frac{\pi}{2}\right)$ when the pendulum is above horizontal.

Several experiments were conducted and some of the obtained transients are shown in the following. The maximum voltage available to the motor was limited to $12 \mathrm{~V}$. For all experiments, the control algorithms were implemented with a sampling period of $2 \mathrm{~ms}$, without transferring continuous designs into discrete time. The pendulum was lifted by hand to a position around vertical and then the control is switched on (on all graphs, the trajectories are shifted, so that as shown, a control action is applied from $t=1 \mathrm{~s}$ on). Both angular speeds were estimated by taking first-order difference from angle measurements.

For the results shown in Fig. 4, the outer-loop controller gains in (10) were designed by pole placement with the desired closed-loop poles being located at -5 , $-7,-9$ and $-11 \mathrm{rad} / \mathrm{s}$. The controller gains were obtained as: $k_{1}=3465, k_{2}=1888$, $k_{3}=374$ and $k_{4}=32$ (N.m)/rad for $k_{1(3)}$ and (N.m.s)/rad for $\left.k_{2(4)}\right)$. As seen in the first place, asymptotic stabilization is not achieved. Asymmetric limit cycle for the pendulum angle is observed, while symmetric cycles establish for both angular speeds, which also guarantees that the motor angle remains bounded. A detailed visualization of the limit cycles is also given in the figures.

In Fig. 5 shows excerpts from experiments, showing the established limit cycles in the pendulum angle and wheel speed trajectories, with poles placed at -3 , $-5,-7$ and $-9 \mathrm{rad} / \mathrm{s}$. The respective controller gains were found to be: $k_{1}=945$, $k_{2}=744, k_{3}=206$ and $k_{4}=24$.
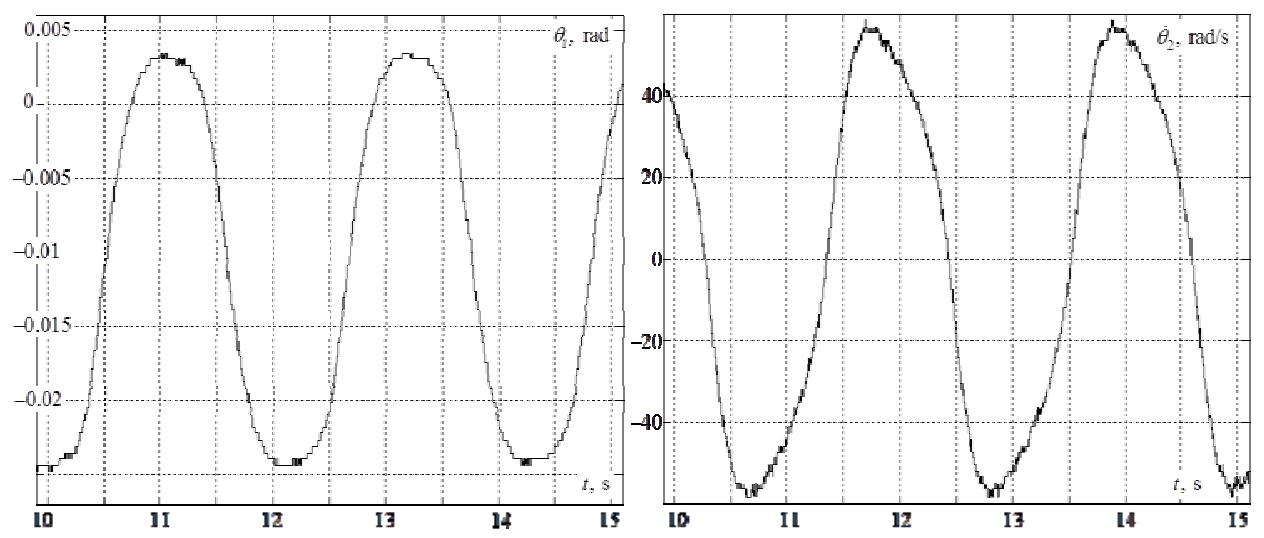

Fig. 5. Established limit cycles of the pendulum angle (to the left) and wheel speed 
It is noted, that again a symmetrical limit cycle of the wheel speed trajectory is observed. By comparing the limit cycles for both cases (Figs. 4 and 5), it is seen that the settings of the outer loop controller influence significantly the parameters of the limit cycles. For the wheel speed trajectory, shown in Fig. 5, a limit cycle with an amplitude of $58 \mathrm{rad} / \mathrm{s}$ and a period of $2.15 \mathrm{~s}$ is observed, while for the case in Fig. 4, the same parameters are with values of $40 \mathrm{rad} / \mathrm{s}$ and $1.22 \mathrm{~s}$ respectively.

In Fig. 6, again the asymptotic behavior of the system is shown, this time with a linearizing control law, designed using the reduced-order model (the control law proposed in [1]). The outer loop state-feedback controller is designed so that the closed-loop poles are placed at $-5,-7$ and $-9 \mathrm{rad} / \mathrm{s}$ and the respective gains are $k_{1}=315, k_{2}=143$ and $k_{4}=21\left(k_{3}=0\right)$. A rather even limit cycle was observed for the pendulum angle, though this was not always the case for the conducted experiments. On the other hand, generally an asymmetrical limit cycle is established of the wheel speed, which in turn results in a diverging wheel angle.

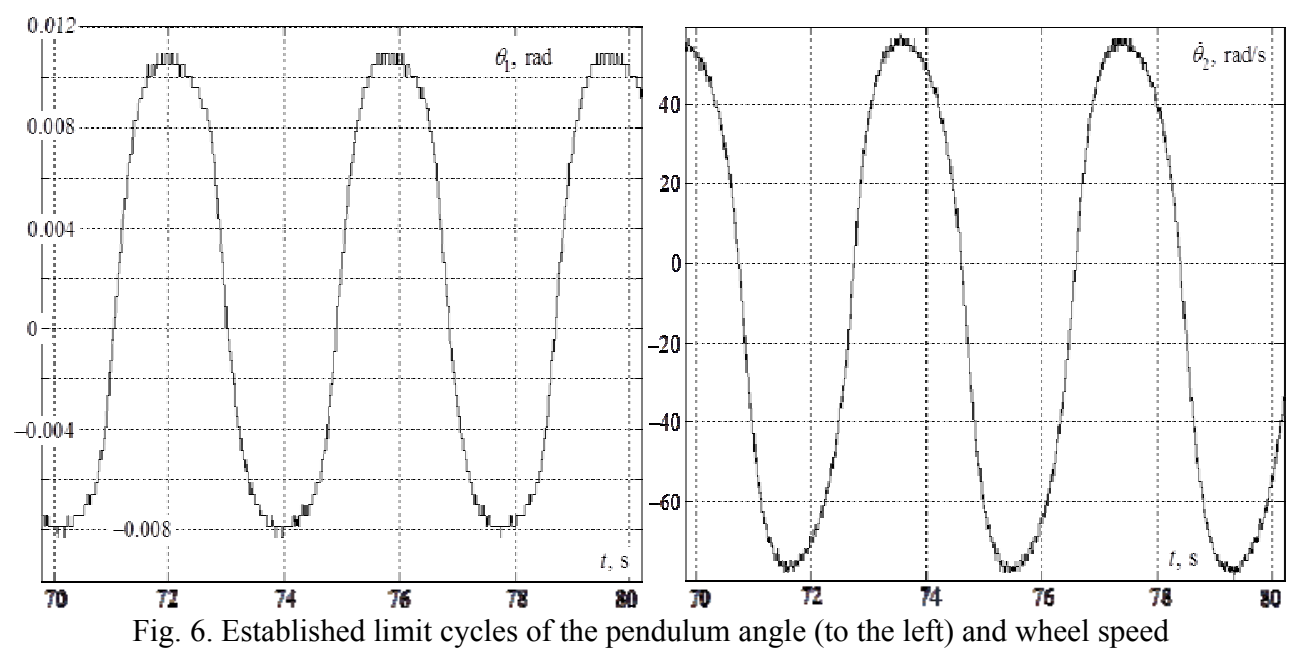

In Fig. 7 transient responses of the system with the input-output linearizing controller in the loop are shown. Since preliminary experiments with a statefeedback controller in the outer loop showed unsatisfactory behavior of the control system, an output feedback controller with integral action was designed and implemented. The input variable to the linearizing controller $-v$ was generated by a discrete-time equivalent of:

$v(s)=k_{0} \frac{(s+4)^{2}}{s(s+40)} X_{1}(s)$, with $k_{0}=-700$, so that the dominating poles of the closed-loop system were placed at around $-10 \pm j 11 \mathrm{rad} / \mathrm{s}$.

As seen, in this case an asymptotic stabilization of the pendulum angle is achieved as it goes to the origin. The wheel speed ultimately settles at around $20 \mathrm{rad} / \mathrm{s}$. An obvious disadvantage of the proposed controller is the fact, that, as the wheel speed grows, the torque generating capabilities of the DC motor diminish, which in turn reduces the ability of the system to reject disturbances. 


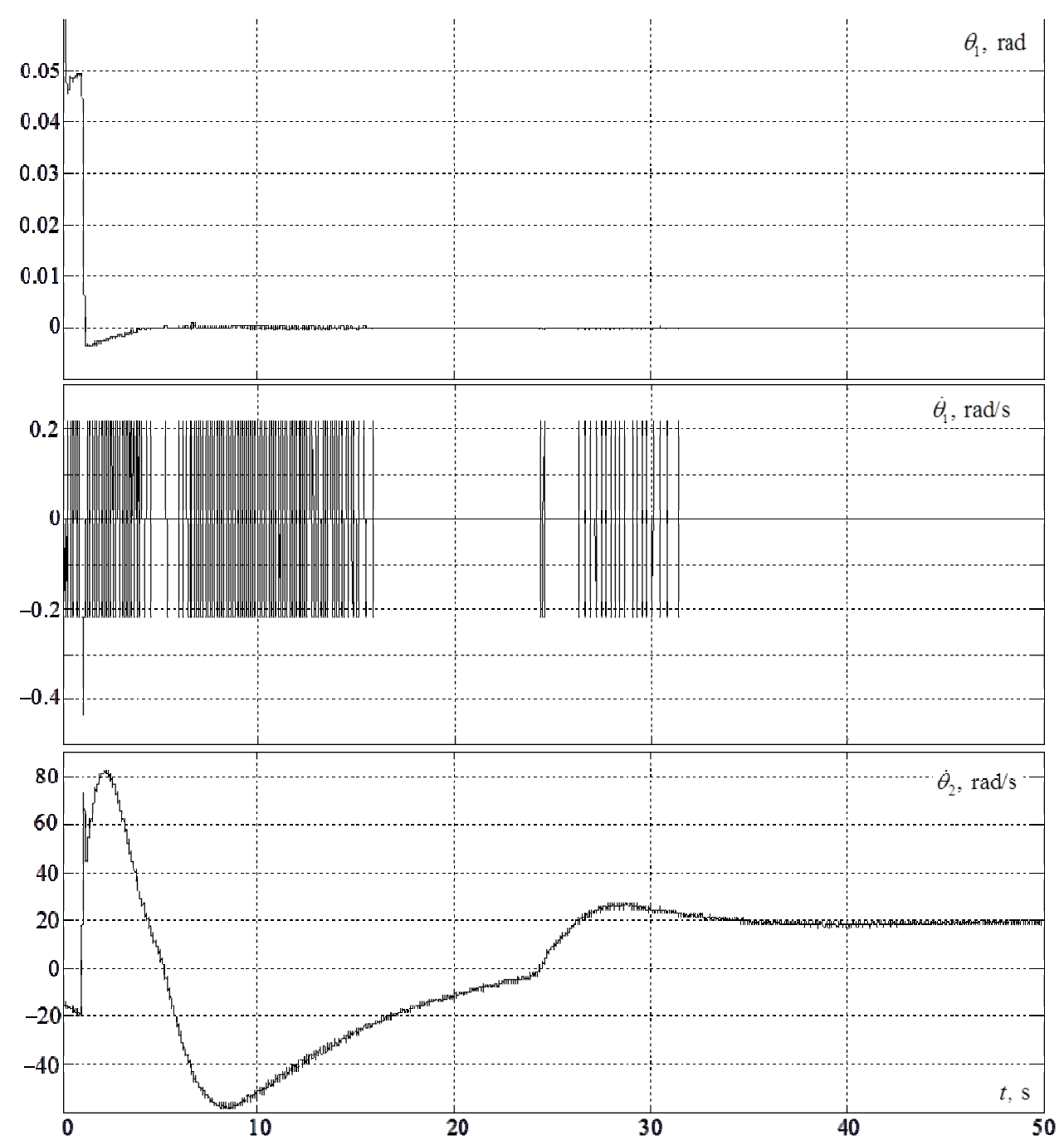

Fig. 7. IWP coordinate trajectories for the input-output linearizing controller

\section{Conclusion}

In this paper two feedback linearizing transformations and control laws are proposed for the stabilization problem of the Inertia Wheel Pendulum at the unstable equilibrium, corresponding to its inverted position. The first controller, leading to a full-state linearization with suitably defined new set of states, represents a generalization of the control law presented in the references, in the sense, that it is based on the full-order model of the system. The experiments on a laboratory test bed are carried out in order to test the suggested performance. It was found that actually no asymptotic stabilization was achieved, but rather stable limit cycles were observed which presumably resulted from the significant friction existing in the base joint of the laboratory apparatus and probably from the 
imperfect compensation of the friction at the DC motor shaft. The parameters of the limit cycles depend on the overall system dynamics, attributed by the outer linear controllers.

Secondly, an input-output linearizing control law is derived, starting from a physically meaningful and directly related to the attacked control problem definition of the system output, being the pendulum angle. The resulting internal dynamics, which is expressed in terms of the wheel speed, is marginally stable. The experimental results have confirmed the expected performance as the pendulum angle settled at the origin, with the wheel speed also settling at a finite value.

\section{References}

1. S p o n g, M. W., P. C o r k e, R. L o z a n o. Nonlinear Control of the Inertia Wheel Pendulum.Automatica, Vol. 37, 2001, 1845-1851.

2. O $1 \mathrm{fat}$ i-S a b e r, R. Global Stabilization of a Flat Underactuated System: The Inertia Wheel Pendulum. - In: Proc. of 40th Conference on Decision and Control, Vol. 4, 2001, 3764-3765.

3. A guil a r-Ib a ne z, C. F., O. O. G. Fri as, M. S. S u a re z C a s t a no n. Stabilization of the Strongly Damping Inertia Wheel Pendulum by a Nested Saturation Functions. - In: Proc. of American Control Conference, 11-13 June 2008, 3434-3439.

4. Ort e g a, R., M. W. S p o n g, F. G o m e z - E s t e r n. Stabilization of Underactuated Mechanical Systems via Interconnection and Damping Assignment. - IEEE Transactions on Automatic Control, Vol. 47, 2002, No 8, 1281-1233.

5. Andary, S., A. Che mori, S. Krut. Control of the Underactuated Inertia Wheel Inverted Pendulum for Stable Limit Cycle Generation. - Advanced Robotics, Vol. 23, 2009, 1999-2014.

6. Ortega, R., E. Garcia-Can s e co. Interconnection and Damping Assignment PassivityBased Control: A Survey. - European Journal of Control, Vol. 10, 2004, 432-450.

7. A s tro m, K. J. Control of Systems with Friction. - In: Proc. of 4th International Conference on Motion and Vibration Control, 1998.

8. S 1 o t i n e, J. J. E., W. L i. Applied Nonlinear Control. Englewood Cliffs. NJ, Prentice Hall, 1991.

9. En e v, S. A Laboratory Inertia Wheel Testbed - Description and First Experiments. - In: Proc. of Technical University of Sofia, Vol. 63, 2013, Issue 1, 89-96. 